\title{
A Family of Boundary Value Methods for Systems of Second-Order Boundary Value Problems
}

\author{
T. A. Biala ${ }^{1}$ and S. N. Jator ${ }^{2}$ \\ ${ }^{1}$ Department of Mathematics and Computer Science, Sule Lamido University, PMB 048, Kafin Hausa, Nigeria \\ ${ }^{2}$ Department of Mathematics and Statistics, Austin Peay State University, Clarksville, TN 37044, USA
}

Correspondence should be addressed to T. A. Biala; bialatoheeb@yahoo.com

Received 10 July 2016; Accepted 13 November 2016; Published 15 January 2017

Academic Editor: Elena Braverman

Copyright (C) 2017 T. A. Biala and S. N. Jator. This is an open access article distributed under the Creative Commons Attribution License, which permits unrestricted use, distribution, and reproduction in any medium, provided the original work is properly cited.

A family of boundary value methods (BVMs) with continuous coefficients is derived and used to obtain methods which are applied via the block unification approach. The methods obtained from these continuous BVMs are weighted the same and are used to simultaneously generate approximations to the exact solution of systems of second-order boundary value problems (BVPs) on the entire interval of integration. The convergence of the methods is analyzed. Numerical experiments were performed to show efficiency and accuracy advantages.

\section{Introduction}

In what follows, we consider the general system of secondorder boundary value problems:

$$
\begin{aligned}
y^{\prime \prime} & =f\left(x, y, y^{\prime}\right), \quad x \in[a, b], \\
y(a) & =y_{0}, \\
y(b) & =y_{N},
\end{aligned}
$$

where $f: \mathbb{R} \times \mathbb{R}^{2 m} \rightarrow \mathbb{R}^{m}$ are continuous functions, $y, y^{\prime}$, and $y^{\prime \prime} \in \mathbb{R}^{m}$, and $m$ is the dimension of the system. These second-order boundary value problems are encountered in several areas of engineering and applied sciences such as celestial mechanics, circuit theory, astrophysics, chemical kinetics, and biology. Most of these problems cannot be solved analytically, thus the need for a numerical approach. In practice, (1) is solved by the multiple shooting technique and the finite difference methods. The construction and implementation of higher order methods for the latter approach are difficult while the former approach suffers from numerical instability if the BVP is stiff [1-3] and singularly perturbed.

In the past few decades, the boundary value methods (BVMs) have been used to solve first-order initial and boundary value problems [4-8]. Their stability and convergence properties have been fully discussed in [5]. These BVMs are also used to solve higher order initial and boundary value problems by first reducing the higher order differential equations into an equivalent first-order system. This approach increases the computational costs and time and also does not utilize additional information associated with specific differential equations such as the oscillatory nature of some solutions $[9,10]$.

Lambert and Watson [11] have derived symmetric schemes for periodic initial value problems of the special second-order $y^{\prime \prime}=f(x, y)$. Brugnano and Trigiante [46] have also derived BVMs for the first-order initial and boundary value problems. Amodio and Iavernaro [12] used BVMs to solve the special second-order problem $y^{\prime \prime}=$ $f(x, y)$. Biala, Biala and Jator, Jator and Li [13-15] applied the BVMs to solve the general second-order problem $y^{\prime \prime}=$ $f\left(x, y, y^{\prime}\right)$ and Aceto et al. [16] constructed symmetric linear multistep methods (LMMs) which were used as BVMs for the special second-order problem $y^{\prime \prime}=f(x, y)$. In this paper, we have derived a class of BVMs and given a general framework via the block unification approach on how to use the BVMs on systems of BVPs for the general second-order differential equations (ODEs). 
The boundary value technique simultaneously generates approximate solution $\left(y_{1}, y_{2}, \ldots, y_{N-1}\right)^{T}$ to the exact solution $\left(y\left(x_{1}\right), y\left(x_{2}\right), \ldots, y\left(x_{N-1}\right)\right)^{T}$ of (1) on the entire interval of integration. The BVMs can only be successfully implemented if used together with appropriate additional methods [5]. In this regard, we have proposed methods which are obtained from the same continuous scheme and are derived via the interpolation and collocation approach [15, 17-19].

The paper is organised as follows. In Section 2, we derive a continuous approximation $U(x)$ of the exact solution $y(x)$. Section 3 gives the specification of the methods. The convergence of the methods is discussed in Section 4. The use and implementation of the methods on ODEs and partial differential equations (PDEs) are detailed in Section 5. Numerical tests and concluding remarks are given in Sections 6 and 7, respectively.

\section{Derivation of Methods}

In this section, we shall use the interpolation and collocation approach [17] to construct a $2 v$-step continuous LMM (CLMM) which will be used to produce the main and additional formulas for solving (1).

Our starting point is to construct the CLMM which has the form

$$
U(x)=\alpha_{\nu}(x) y_{n+\nu}+\alpha_{0}(x) y_{n}+h^{2} \sum_{i=0}^{2 v} \beta_{i}(x) f_{n+i},
$$

where $\alpha_{0}(x), \alpha_{\nu}(x)$, and $\beta_{i}(x)$ are continuous coefficients and $v$ is chosen to be half the step number so that each formula, derived from (2), satisfies the root condition. The main and additional methods are then obtained by evaluating (2) at $x_{n+j}(j=1(1) 2 v, j \neq \nu)$ to obtain the formulas of the form

$$
\begin{gathered}
y_{n+j}+2 y_{n+v}-y_{n}=h^{2} \sum_{i=0}^{2 v} \beta_{i} f_{n+i}, \\
j=1, \ldots, v-1, v+1, \ldots, 2 v, \\
h y_{n+k}^{\prime}+\alpha_{\nu}^{\prime} y_{n+v}+\alpha_{0}^{\prime} y_{n}=h^{2} \sum_{i=0}^{2 v} \beta_{i}^{\prime} f_{n+i}, \quad k=0(1)(2 v)
\end{gathered}
$$

obtained from the first derivative of (2).

Next, we discuss the construction of (2) in the theorem that follows.

Theorem 1. Let (2) satisfy the following equations:

$$
\begin{gathered}
U\left(x_{n+r}\right)=y_{n+r} \quad r=0, \nu, \\
U^{\prime \prime}\left(x_{n+i}\right)=f_{n+i} \quad i=0(1)(2 \nu) .
\end{gathered}
$$

Then, the continuous representation (2) is equivalent to

$$
U(x)=\sum_{j=0}^{2 v+2} \frac{\operatorname{det}\left(V_{j}\right)}{\operatorname{det}(V)} P_{j}(x),
$$

where one defines the matrix $V$ as

$$
V=\left(\begin{array}{cccc}
P_{0}\left(x_{n}\right) & P_{1}\left(x_{n}\right) & \cdots & P_{2 v+2}\left(x_{n}\right) \\
P_{0}\left(x_{n+v}\right) & P_{1}\left(x_{n+v}\right) & \cdots & P_{2 v+2}\left(x_{n+v}\right) \\
P_{0}^{\prime \prime}\left(x_{n}\right) & P_{1}^{\prime \prime}\left(x_{n}\right) & \cdots & P_{2 v+2}^{\prime \prime}\left(x_{n}\right) \\
P_{0}^{\prime \prime}\left(x_{n+1}\right) & P_{1}^{\prime \prime}\left(x_{n+1}\right) & \cdots & P_{2 v+2}^{\prime \prime}\left(x_{n+1}\right) \\
\vdots & \vdots & \vdots & \vdots \\
P_{0}^{\prime \prime}\left(x_{n+2 v}\right) & P_{1}^{\prime \prime}\left(x_{n+2 v}\right) & \cdots & P_{2 v+2}^{\prime \prime}\left(x_{n+2 v}\right)
\end{array}\right),
$$

$V_{j}$ is obtained by replacing the jth column of $V$ by

$$
W=\left(y_{n}, y_{n+v}, f_{n}, f_{n+1}, \ldots, f_{n+2 v}\right)^{T},
$$

and $P_{j}(x)=x^{j}, j=0(1)(2 v+2)$ are basis functions.

Proof. We require that method (2) be defined by the assumed polynomial basis functions

$$
\begin{aligned}
\alpha_{j}(x) & =\sum_{i=0}^{2 v+2} \alpha_{i+1, j} P_{i}(x), \quad j=0, v \\
h^{2} \beta_{j}(x) & =\sum_{i=0}^{2 v+2} h^{2} \beta_{i+1, j} P_{i}(x), \quad j=0(1)(2 v),
\end{aligned}
$$

where $\alpha_{i+1, j}$ and $h^{2} \beta_{i+1, j}$ are coefficients to be determined.

Substituting (9) into (2), we have

$$
\begin{aligned}
U(x)= & \sum_{i=0}^{2 v+2} \alpha_{i+1,0} P_{i}(x) y_{n}+\sum_{i=0}^{2 v+2} \alpha_{i+1, \nu} P_{i}(x) y_{n+\nu} \\
& +\sum_{j=0}^{2 v} \sum_{i=0}^{2 v+2} h^{2} \beta_{i+1, j} P_{i}(x) f_{n+j}
\end{aligned}
$$

which is simplified to

$$
\begin{aligned}
& U(x) \\
& =\sum_{i=0}^{2 v+2}\left\{\alpha_{i+1,0} y_{n}+\alpha_{i+1, \nu} y_{n+\nu}+\sum_{j=0}^{2 v} h^{2} \beta_{i+1, j} f_{n+j}\right\} P_{i}(x)
\end{aligned}
$$

and expressed in the form

$$
U(x)=\sum_{i=0}^{2 v+2} \tau_{i} P_{i}(x)
$$

where

$$
\tau_{i}=\alpha_{i+1,0} y_{n}+\alpha_{i+1, \nu} y_{n+\nu}+\sum_{j=0}^{2 \nu} h^{2} \beta_{i+1, j} f_{n+j}
$$

Imposing conditions (5) on (12), we obtain a system of $(2 v+3)$ equations which can be expressed as $V L=W$, where 
$L=\left(\tau_{0}, \tau_{1}, \ldots, \tau_{2 v+2}\right)^{T}$ is a vector of $(2 \nu+3)$ undetermined coefficients.

Using Crammer's rule, the elements of $L$ are determined and given as

$$
\tau_{i}=\frac{\operatorname{det}\left(V_{j}\right)}{\operatorname{det}(V)}, \quad j=0(1)(2 v+2)
$$

where $V_{j}$ is obtained by replacing the $j$ th column of $V$ by $W$. We rewrite (12) using the newly found elements of $L$ as in (6); that is,

$$
U(x)=\sum_{j=0}^{2 v+2} \frac{\operatorname{det}\left(V_{j}\right)}{\operatorname{det}(V)} P_{j}(x) .
$$

\section{Specification of Methods}

In this section, we specify the family of methods by evaluating the CLMM (2) at $x_{n+i}, i=i, v-1, v+1, \ldots, 2 v$, which is also used to obtain the derivative formula given by

$$
\begin{aligned}
& U^{\prime}(x) \\
& \quad=\frac{1}{h}\left(\alpha_{\nu}^{\prime}(x) y_{n+v}+\alpha_{0}^{\prime}(x) y_{n}+h^{2} \sum_{i=0}^{2 v} \beta_{i}^{\prime}(x) f_{n+i}\right),
\end{aligned}
$$

which is effectively applied by imposing that

$$
\begin{aligned}
& U^{\prime}(a)=y_{0}^{\prime}, \\
& U^{\prime}(b)=y_{N}^{\prime},
\end{aligned}
$$

to produce derivative formulas of the form (4).

3.1. BVM of Orders 4, 6, and 8. For $v=1$, the BVM of order 4 is given as follows (where we have denoted a BVM with $k$ step number as BVMk):

BVM2

$$
y_{n+2}-2 y_{n+1}+y_{n}=\frac{h^{2}}{12}\left(f_{n}+10 f_{n+1}+f_{n+2}\right) \text {, }
$$

with the derivative formulas

$$
\begin{aligned}
h y_{n}^{\prime}-y_{n+1}+y_{n} & =\frac{h^{2}}{24}\left(-7 f_{n}-6 f_{n+1}+f_{n+2}\right), \\
h y_{n+1}^{\prime}-y_{n+1}+y_{n} & =\frac{h^{2}}{24}\left(3 f_{n}+10 f_{n+1}-f_{n+2}\right), \\
h y_{n+2}^{\prime}-y_{n+1}+y_{n} & =\frac{h^{2}}{24}\left(f_{n}+26 f_{n+1}+9 f_{n+2}\right) .
\end{aligned}
$$

For $v=2$, we obtain the BVM of order 6 given as follows:

BVM4

$$
\begin{aligned}
& y_{n+1}-\frac{1}{2} y_{n+2}-\frac{1}{2} y_{n}=\frac{h^{2}}{480}\left(-19 f_{n}-204 f_{n+1}\right. \\
& \left.-14 f_{n+2}-4 f_{n+3}+f_{n+4}\right), \\
& y_{n+3}-\frac{3}{2} y_{n+2}+\frac{1}{2} y_{n}=\frac{h^{2}}{480}\left(17 f_{n}+252 f_{n+1}\right. \\
& \left.+402 f_{n+2}+52 f_{n+3}-3 f_{n+4}\right), \\
& y_{n+4}-2 y_{n+2}+y_{n}=\frac{h^{2}}{15}\left(f_{n}+16 f_{n+1}+26 f_{n+2}\right. \\
& \left.+16 f_{n+3}+f_{n+4}\right),
\end{aligned}
$$

$$
n=0(4)(N-4)
$$

with the derivative formulas

$$
\begin{aligned}
& h y_{n}^{\prime}-\frac{1}{2} y_{n+2}+\frac{1}{2} y_{n}=\frac{h^{2}}{180}\left(-53 f_{n}-144 f_{n+1}\right. \\
& \left.+30 f_{n+2}-16 f_{n+3}+3 f_{n+4}\right), \\
& h y_{n+1}^{\prime}-\frac{1}{2} y_{n+2}+\frac{1}{2} y_{n}=\frac{h^{2}}{720}\left(39 f_{n}+70 f_{n+1}\right. \\
& \left.\quad-144 f_{n+2}+42 f_{n+3}-7 f_{n+4}\right), \\
& h y_{n+2}^{\prime}-\frac{1}{2} y_{n+2}+\frac{1}{2} y_{n}=\frac{h^{2}}{180}\left(5 f_{n}+104 f_{n+1}+78 f_{n+2}\right. \\
& \left.\quad-8 f_{n+3}+f_{n+4}\right),
\end{aligned}
$$

$$
\begin{aligned}
& h y_{n+3}^{\prime}-\frac{1}{2} y_{n+2}+\frac{1}{2} y_{n}=\frac{h^{2}}{720}\left(31 f_{n}+342 f_{n+1}\right. \\
& \left.\quad+768 f_{n+2}+314 f_{n+3}-15 f_{n+4}\right), \\
& h y_{n+4}^{\prime}-\frac{1}{2} y_{n+2}+\frac{1}{2} y_{n}=\frac{h^{2}}{180}\left(3 f_{n}+112 f_{n+1}+56 f_{n+2}\right. \\
& \left.\quad+240 f_{n+3}+59 f_{n+4}\right) .
\end{aligned}
$$

For $v=3$, we obtain the BVM of order 8 given as follows: BVM6

$$
\begin{aligned}
& y_{n+1}-\frac{1}{3} y_{n+3}-\frac{2}{3} y_{n}=\frac{h^{2}}{60480}\left(-2803 f_{n}-37950 f_{n+1}\right. \\
& -14913 f_{n+2}-7108 f_{n+3}+3147 f_{n+4}-990 f_{n+5} \\
& \left.\quad+137 f_{n+6}\right),
\end{aligned}
$$




$$
\begin{aligned}
& y_{n+2}-\frac{2}{3} y_{n+3}-\frac{1}{3} y_{n}=\frac{h^{2}}{60480}\left(-1291 f_{n}-21906 f_{n+1}\right. \\
& -32133 f_{n+2}-6288 f_{n+3}+1467 f_{n+4}-402 f_{n+5} \\
& \left.+53 f_{n+6}\right) \text {, } \\
& y_{n+4}-\frac{4}{3} y_{n+3}+\frac{1}{3} y_{n}=\frac{h^{2}}{30240}\left(661 f_{n}+10734 f_{n+1}\right. \\
& +19323 f_{n+2}+27268 f_{n+3}+2523 f_{n+4}-18 f_{n+5} \\
& \left.-11 f_{n+6}\right) \text {, } \\
& y_{n+5}-\frac{5}{3} y_{n+3}+\frac{2}{3} y_{n}=\frac{h^{2}}{12096}\left(535 f_{n}+8550 f_{n+1}\right. \\
& +15501 f_{n+2}+22900 f_{n+3}+11889 f_{n+4}+1158 f_{n+5} \\
& \left.-53 f_{n+6}\right) \\
& y_{n+6}-2 y_{n+3}+y_{n}=\frac{h^{2}}{2240}\left(141 f_{n}+2430 f_{n+1}\right. \\
& +4131 f_{n+2}+6756 f_{n+3}+4131 f_{n+4}+2430 f_{n+5} \\
& \left.+141 f_{n+6}\right) \text {, }
\end{aligned}
$$$$
n=0(6)(N-6) \text {, }
$$

with the derivatives

$$
\begin{aligned}
& h y_{n}^{\prime}-\frac{1}{3} y_{n+3}+\frac{1}{3} y_{n}=\frac{h^{2}}{13440}\left(-3795 f_{n}-14850 f_{n+1}\right. \\
& \quad+2403 f_{n+2}-6300 f_{n+3}+3267 f_{n+4}-1026 f_{n+5} \\
& \left.\quad+141 f_{n+6}\right), \\
& h y_{n+1}^{\prime}-\frac{1}{3} y_{n+3}+\frac{1}{3} y_{n}=\frac{h^{2}}{120960}\left(4019 f_{n}-3426 f_{n+1}\right. \\
& \quad-7125 f_{n+2}+18308 f_{n+3}-11019 f_{n+4}+3390 f_{n+5} \\
& \left.\quad-457 f_{n+6}\right), \\
& \quad h y_{n+2}^{\prime}-\frac{1}{3} y_{n+3}+\frac{1}{3} y_{n}=\frac{h^{2}}{120960}\left(2293 f_{n}\right. \\
& \quad+46830 f_{n+1}+22683 f_{n+2}-14204 f_{n+3} \\
& \left.\quad+3579 f_{n+4}-786 f_{n+5}+85 f_{n+6}\right) \\
& \quad+9369 f_{n+2}+6576 f_{n+3}-1107 f_{n+4}+270 f_{n+5} \\
& h y_{n+3}^{\prime}-\frac{1}{3} y_{n+3}+\frac{1}{3} y_{n}=\frac{h^{2}}{13440}\left(315 f_{n}+4590 f_{n+1}\right.
\end{aligned}
$$

$$
\begin{aligned}
& h y_{n+4}^{\prime}-\frac{1}{3} y_{n+3}+\frac{1}{3} y_{n}=\frac{h^{2}}{120960}\left(2453 f_{n}\right. \\
& \quad+44526 f_{n+1}+70779 f_{n+2}+135812 f_{n+3} \\
& \left.\quad+51675 f_{n+4}-3090 f_{n+5}+245 f_{n+6}\right) \\
& h y_{n+5}^{\prime}-\frac{1}{3} y_{n+3}+\frac{1}{3} y_{n}=\frac{h^{2}}{120960}\left(2995 f_{n}\right. \\
& \quad+40350 f_{n+1}+85377 f_{n+2}+103300 f_{n+3} \\
& \left.\quad+145653 f_{n+4}+47166 f_{n+5}-1481 f_{n+6}\right) \\
& h y_{n+6}^{\prime}-\frac{1}{3} y_{n+3}+\frac{1}{3} y_{n}=\frac{h^{2}}{13440}\left(141 f_{n}+5886 f_{n+1}\right. \\
& \quad+4995 f_{n+2}+19812 f_{n+3}+5859 f_{n+4}+19710 f_{n+5} \\
& \left.\quad+4077 f_{n+6}\right),
\end{aligned}
$$

\section{Convergence of the Methods}

In this section, we shall establish the convergence of the BVMs derived in the previous section. We emphasize that we evaluate $(2)$ at $x_{n+1}, x_{n+2}, \ldots, x_{n+v-1}, x_{n+v+1}, \ldots, x_{n+2 v}$ to obtain

$$
\begin{gathered}
y_{n+1}+\alpha_{\nu}^{(1)} y_{n+\nu}+\alpha_{0}^{(1)} y_{n}=h^{2} \sum_{i=0}^{2 v} \beta_{i}^{(1)} f_{n+i} \\
y_{n+2}+\alpha_{\nu}^{(2)} y_{n+\nu}+\alpha_{0}^{(2)} y_{n}=h^{2} \sum_{i=0}^{2 v} \beta_{i}^{(2)} f_{n+i} \\
\vdots \\
y_{n+\nu-1}+\alpha_{v}^{(\nu-1)} y_{n+\nu}+\alpha_{0}^{(\nu-1)} y_{n}=h^{2} \sum_{i=0}^{2 v} \beta_{i}^{(\nu-1)} f_{n+i} \\
y_{n+\nu+1}+\alpha_{\nu}^{(\nu+1)} y_{n+\nu}+\alpha_{0}^{(\nu+1)} y_{n}=h^{2} \sum_{i=0}^{2 v} \beta_{i}^{(\nu+1)} f_{n+i} \\
\vdots \\
y_{n+2 v}+\alpha_{v}^{(2 v)} y_{n+\nu}+\alpha_{0}^{(2 v)} y_{n}=h^{2} \sum_{i=0}^{2 v} \beta_{i}^{(2 \nu)} f_{n+i}
\end{gathered}
$$

and also evaluate $U^{\prime}(x)$ at $x_{n+i}, i=0(1)(2 v)$, to obtain

$$
\begin{gathered}
h y_{n}^{\prime}+\alpha_{v}^{\prime(0)} y_{n+v}+\alpha_{0}^{\prime(0)} y_{n}=h^{2} \sum_{i=0}^{2 v} \beta_{i}^{\prime(0)} f_{n+i} \\
h y_{n+1}^{\prime}+\alpha_{v}^{\prime(1)} y_{n+v}+\alpha_{0}^{\prime(1)} y_{n}=h^{2} \sum_{i=0}^{2 v} \beta_{i}^{\prime(1)} f_{n+i}
\end{gathered}
$$




$$
h y_{n+2 v}^{\prime}+\alpha_{v}^{\prime(2 v)} y_{n+v}+\alpha_{0}^{\prime(2 v)} y_{n}=h^{2} \sum_{i=0}^{2 v} \beta_{i}^{\prime(2 v)} f_{n+i}
$$

We note that the formulas in (24) and 4 are $O\left(h^{2 v+4}\right)$.

We introduce the matrices $P$ and $Q$ such that systems (24) and 4 are given by

$$
P \bar{Y}-Q F(\bar{Y})+C=0
$$

and the exact form of the system is

$$
P Y-Q F(Y)+C+L(h)=0,
$$

where

$$
\begin{gathered}
P=\left[\begin{array}{c|c}
P_{11} & \mathbf{O} \\
\hline P_{21} & P_{22}
\end{array}\right], \\
Q=\left[\begin{array}{c|c}
Q_{11} & \mathbf{O} \\
\hline Q_{21} & \mathbf{O}
\end{array}\right] .
\end{gathered}
$$

$P_{i j}$ and $Q_{i j}$ are $N \times N$ matrices, $\mathbf{O}$ is the zero matrix, $\bar{Y}=\left(h y_{0}^{\prime}, y_{1}, \ldots, y_{N-1}, h y_{1}^{\prime}, \ldots, h y_{N}^{\prime}\right)^{T}, F=$ $\left(h f_{0}^{\prime}, f_{1}, \ldots, f_{N-1}, h f_{1}^{\prime}, \ldots, h f_{N}^{\prime}\right)^{T}, C$ is a vector of constants, and $L(h)$ is the truncation error vector of the formulas in (24) and 4.

Lemma 2. Let $P$ be a $2 \times 2$ block lower triangular matrix given by

$$
P=\left[\begin{array}{c|c}
P_{11} & \mathbf{O} \\
\hline P_{21} & P_{22}
\end{array}\right],
$$

where each submatrix is of order $N$ and $\mathbf{O}$ is the zero matrix. Then, $P$ is invertible if and only if $P_{11}$ and $P_{22}$ are invertible. Moreover,

$$
P^{-1}=\left[\begin{array}{c|c}
P_{11}^{-1} & \mathbf{O} \\
\hline-P_{22}^{-1} P_{21} P_{11}^{-1} & P_{22}^{-1}
\end{array}\right] .
$$

$P_{22}$ is an identity matrix so that $\left\|P_{22}^{-1}\right\|=1$. Thus, to obtain an estimate for $\left\|P^{-1}\right\|$, it suffices to show the existence of the inverse of $P_{11}$.

Now, we define

$$
\bar{P}_{11}=D_{11}-P_{11}
$$

where $D_{11}=\operatorname{diag}\left(P_{11}\right)$ so that

$$
D_{11}^{-1} P_{11}=I-D_{11}^{-1} \bar{P}_{11}
$$

and consequently $P_{11}$ is nonsingular provided $\rho\left(D_{11}^{-1} \bar{P}_{11}\right)<1$ ([21]).

Thus, $P^{-1}$ exists provided $\rho\left(D_{11}^{-1} \bar{P}_{11}\right)<1$.

Lemma 3. If $H<1 / h^{2}\left\|P^{-1}\right\|\|Q\|$, then the matrix $A=$ $P-h^{2} Q J$ is monotone, that is $A^{-1}>0$, where $J$ is also a $2 \times 2$ block matrix of first partial derivatives and $H=$ $\max \left\{\left|\partial f_{i} / \partial y_{i}\right|,\left|\partial f_{i} / \partial y_{i}^{\prime}\right|, \quad i=1(1) N\right\}$.
Proof.

$$
\begin{aligned}
& \begin{aligned}
A & =P-h^{2} Q J \\
A P^{-1} & =I-h^{2} Q J P^{-1} \\
P A^{-1} & =\left(I-h^{2} Q J P^{-1}\right)^{-1}=I+\left(h^{2} Q J P^{-1}\right) \\
& +\left(h^{2} Q J P^{-1}\right)^{2}+\left(h^{2} Q J P^{-1}\right)^{3}+\cdots \\
& =\left[I+h^{2} Q J P^{-1}\right] \\
& \cdot\left[I+\left(h^{2} Q J P^{-1}\right)^{2}+\left(h^{2} Q J P^{-1}\right)^{4}+\cdots\right] .
\end{aligned}
\end{aligned}
$$

The two series converge provided the spectral radius $\rho\left(h^{2} Q J P^{-1}\right)<1$ :

$$
\begin{aligned}
A^{-1}= & {\left[P^{-1}+h^{2} P^{-1} Q J P^{-1}\right] } \\
& \cdot\left[I+\left(h^{2} Q J P^{-1}\right)^{2}+\left(h^{2} Q J P^{-1}\right)^{4}+\cdots\right] .
\end{aligned}
$$

The infinite series is nonnegative. Thus, to show that $A$ is monotone, it suffices to show that

$$
\begin{aligned}
P^{-1}+h^{2} P^{-1} Q J P^{-1} & >0 \\
P^{-1} & >h^{2} P^{-1} Q J P^{-1} \\
I & >h^{2} P^{-1} Q J \\
\left\|h^{2} P^{-1} Q J\right\| & \leq h^{2}\left\|P^{-1}\right\|\|Q\|\|J\|<1
\end{aligned}
$$

for $\|J\|=H<1 / h^{2}\left\|P^{-1}\right\|\|Q\|$.

Theorem 4. Let $\bar{Y}$ be an approximation of the solution vector $Y$ for the system obtained on a partition $\pi_{N}:=\left\{a=x_{0}<\right.$ $\left.x_{1}<x_{2}<\cdots<x_{m-1}<x_{m}=b\right\}$ from systems (24) and 4. If $e_{i}=\left|y_{i}-y\left(x_{i}\right)\right|$ and $e_{i}^{\prime}=\left|y_{i}^{\prime}-y^{\prime}\left(x_{i}\right)\right|$, where the exact solution $y(x)$ is assumed to be several times differentiable on $[a, b]$, and if $\|E\|=\|Y-\bar{Y}\|$, then, for sufficiently small $h,\|E\|=O\left(h^{2 v+2}\right)$.

Proof. Subtracting (27) from (26), we obtain

$$
A E=L(h)
$$

Under the conditions of Lemma $3, A^{-1}$ exists and is nonnegative. Therefore,

$$
\begin{aligned}
E & =\left(P-h^{2} Q J\right)^{-1} L(h) \\
& =\left(I-h^{2} P^{-1} Q J\right)^{-1} P^{-1} L(h) \\
\|E\| & \leq\left\|\left(I-h^{2} P^{-1} Q J\right)^{-1}\right\|\left\|P^{-1}\right\|\|L(h)\| \\
& \leq \frac{\left\|P^{-1}\right\|\|L(h)\|}{1-h^{2}\left\|P^{-1}\right\|\|Q\|\|J\|}
\end{aligned}
$$


provided $h^{2}\left\|P^{-1}\right\|\|Q\|\|J\|<1$. Hence,

$$
\|E\| \leq \frac{\left\|P^{-1}\right\| O\left(h^{2 \nu+4}\right)}{1-h^{2} H\left\|P^{-1}\right\|\|Q\|}=O\left(h^{2 \nu+2}\right) .
$$

\section{Use of Methods}

In this section, we discuss the use of methods in (16) and (17) for $n=0(2 v)(N-2 v)$, where $N$ is a multiple of $2 v$. We emphasize that the methods in (16) and (17) are all main methods since they are weighted the same and their use leads to a single matrix equation which can be solved for the unknowns. For example, for BVM6, we make use of each of the methods above in steps of 6 ; that is, $n=0,6, \ldots, N-6$. This results in a system of $2 \mathrm{~N}$ equations in $2 \mathrm{~N}$ unknowns which can be easily solved for the unknowns. Below is an algorithm for the use of the methods.

The methods are implemented as BVMs by efficiently using the following steps.

Step 1. Use the methods in (16) and (17) for $n=0$ to obtain $\mathbf{Y}_{1}$ in the interval $\left[y_{0}, y_{2 v}\right]$ and for $n=1 \mathbf{Y}_{2}$ is obtained in the interval $\left[y_{2 v}, y_{4 v}\right]$. Similarly, for $n=2,3, \ldots,(\Gamma-1)$, we obtain $\mathbf{Y}_{3}, \ldots, \mathbf{Y}_{\Gamma}$, where $N=2 v \times \Gamma$ in the intervals, $\left[y_{4 v}, y_{6 v}\right],\left[y_{6 v}, y_{8 v}\right], \ldots,\left[y_{N-2 v}, y_{N}\right]$, respectively.

Step 2. The unified block given by the system $\mathbf{Y}_{1} \cup \mathbf{Y}_{2} \cup \cdots \cup \mathbf{Y}_{\Gamma-1} \cup \mathbf{Y}_{\Gamma}$ obtained in step 1 results in a system of $2 N$ equations in $2 N$ unknowns which can be easily solved.

Step 3. The values of the solution and the first derivatives of (1) are generated by the sequence $\left\{y_{n}\right\},\left\{y_{n}^{\prime}\right\}, n=0, \ldots, N$, obtained as the solution in Step 2.

We note that all computations were carried out in Mathematica 10.0 enhanced by the feature FindRoot[ ].

\section{Numerical Examples}

In this section, we consider seven numerical examples. Examples 1 to 5 were solved using the BVMs $k=4, k=$ 6 , and $k=8$ (derived in this paper) of orders 6,8 , and 10 , respectively. Also, these examples were solved using the Extended Trapezoidal Methods of the second kind (ETRs) and the Top Order Methods (TOMs) given in [5] of orders 6 and 10, respectively. Comparisons are made between the BVM $k=4$ and the ETRs [5] as well as between the BVM $k=8$ and the TOMs [5] by obtaining the maximum errors $E y$ in the interval of integration. We also compared our methods with the Sinc-Collocation method [20]. Examples 6 and 7 were solved using the BVMs of order 6 . We note that the number of function evaluations (NFEs) involved in implementing the BVMs is $N \times 2 v$ in the entire range of integration. The code was based on Newton's method which uses the feature FindRoot[ ] or NSolve[ ] for linear problems in Mathematica. The efficiency curves show the plot of the logarithm of Ey against the number of function evaluations for each method.

Example 1. We consider the linear system of second-order boundary value problems given in [20]

$$
\begin{aligned}
& \frac{d^{2} y_{1}}{d x^{2}}+(2 x-1) \frac{d y_{1}}{d x}+\cos (\pi x) \frac{d y_{2}}{d x}=f_{1}(x), \\
& \quad 0<x<1 \\
& \frac{d^{2} y_{2}}{d x^{2}}+x y_{1}=f_{2}(x) \\
& y_{1}(0)=y_{2}(0)=y_{1}(1)=y_{2}(1)=0,
\end{aligned}
$$

where

$$
\begin{aligned}
f_{1}(x)= & -\pi^{2} \sin (\pi x) \\
& +(2 x-1)(\pi+1) \cos (\pi x), \\
f_{2}(x)= & 2+x \sin (\pi x) \\
\text { Exact: } y_{1}(x)= & \sin (\pi x), \\
y_{2}(x)= & x^{2}-x .
\end{aligned}
$$

This problem was solved using the ETRs and BVM of order 6 as well as the TOMs and BVM of order 10. The maximum Euclidean norm of the absolute errors in $y_{1}$ and $y_{2}$ was obtained in the entire interval of integration. In Table 1, we compared the Sinc-Collocation method [20] with the BVM of order 8 . Table 2 shows the comparison between the ETRs, BVM4, TOMs, and BVM8. While the results of these methods are of approximate accuracy, we emphasize that the TOMs and ETRs use 20 function evaluations per step while the BVM4 and BVM8 use $8 N$ and $16 N$ function evaluations for this system. Hence, the BVMs are quite accurate and efficient. We also calculated the Rate of Convergence (ROC) using the formula $\log _{2}\left(E^{2 h} / E^{h}\right)$, where $E^{h}$ is the error obtained using step size $h$. The ROC of the BVM4 and ETRs shows that these methods are consistent with the theoretical order (order 6) behavior of the methods. We omit the ROC of the TOMs and BVM8 because their errors are mainly due to round-off errors rather than to truncation errors. Figure 1 also shows the efficiency curves of these methods.

Example 2. Consider the nonlinear BVP given in [22]

$$
\begin{aligned}
& \frac{d^{2} y_{1}}{d x^{2}}+x y_{1}+2 x y_{2}+x y_{1}^{2}=f_{1}(x), \quad 0<x<1 \\
& \frac{d^{2} y_{2}}{d x^{2}}+y_{2}+x^{2} y_{1}+\sin (x) y_{2}^{2}=f_{2}(x) \\
& y_{1}(0)=y_{2}(0)=y_{1}(1)=y_{2}(1)=0,
\end{aligned}
$$

where

$$
\begin{aligned}
f_{1}(x)= & -2+x\left(x-x^{2}\right)+x\left(x-x^{2}\right)^{2} \\
& -2 x \sin (\pi x)
\end{aligned}
$$


TABle 1: Maximum errors for Example 1.

\begin{tabular}{lccccc}
\hline & Sinc-Coll. [20] & & \multicolumn{2}{c}{ BVM6 } \\
$N$ & $E y_{1}$ & $E y_{2}$ & $N$ & $E y_{1}$ \\
\hline 20 & $3.128 e-05$ & $1.175 e-06$ & 18 & $5.309 e-09$ & $2.853 e-10$ \\
40 & $1.829 e-07$ & $5.095 e-09$ & 36 & $2.332 e-11$ & $1.154 e-12$ \\
60 & $3.573 e-09$ & $7.696 e-11$ & 54 & $8.883 e-13$ & $4.535 e-14$ \\
80 & $1.287 e-10$ & $2.267 e-11$ & 78 & $4.763 e-14$ & $1.915 e-15$ \\
100 & $6.389 e-12$ & $1.026 e-13$ & 96 & $9.326 e-15$ & $1.388 e-16$ \\
\hline
\end{tabular}

TABle 2: Maximum Errors for different stepsizes for Example 1.

\begin{tabular}{|c|c|c|c|c|c|c|}
\hline$N$ & ETRs [5] & ROC & BVM4 & ROC & TOMs [5] & BVM8 \\
\hline 20 & $7.281 e-8$ & & $1.391 e-7$ & & $5.736 e-11$ & $6.952 e-12$ \\
\hline 40 & $1.165 e-9$ & 5.96 & $2.267 e-9$ & 5.94 & $1.958 e-14$ & $4.514 e-14$ \\
\hline 80 & $2.023 e-11$ & 5.86 & $3.530 e-11$ & 6.01 & $3.818 e-16$ & $3.856 e-16$ \\
\hline 160 & $3.325 e-13$ & 5.93 & $5.476 e-13$ & 6.01 & $4.578 e-16$ & $6.621 e-16$ \\
\hline
\end{tabular}

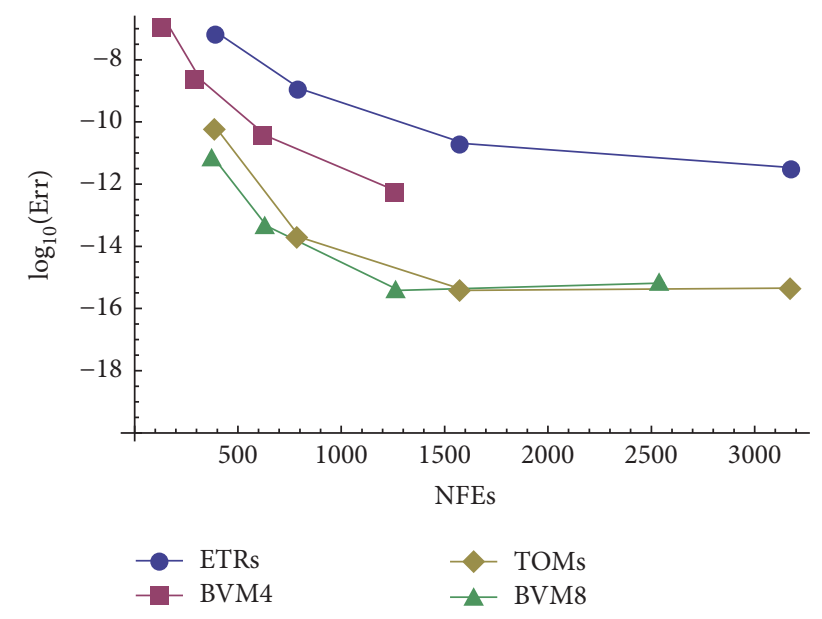

Figure 1: Efficiency curve for Example 1.

$$
\begin{aligned}
f_{2}(x)= & x^{3}(1-x) \\
& +\sin (\pi x)(1+\sin (x) \sin (\pi x)) \\
& -\pi^{2} \sin (\pi x)
\end{aligned}
$$

Exact: $y_{1}(x)=x-x^{2}$

$$
y_{2}(x)=\sin (\pi x) \text {. }
$$

The maximum Euclidean norm of the absolute errors in $y_{1}$ and $y_{2}$ was obtained in the range of integration. Table 3 shows the comparison between the ETRs, BVM4, TOMs, and BVM8. While the results of these methods are of approximate accuracy, we emphasize that the TOMs and ETRs use 20 function evaluations per step while the BVM4 and BVM8 use $8 \mathrm{~N}$ and $16 \mathrm{~N}$ function evaluations for this system. We also calculated the ROC of the BVM4 and ETRs which shows that

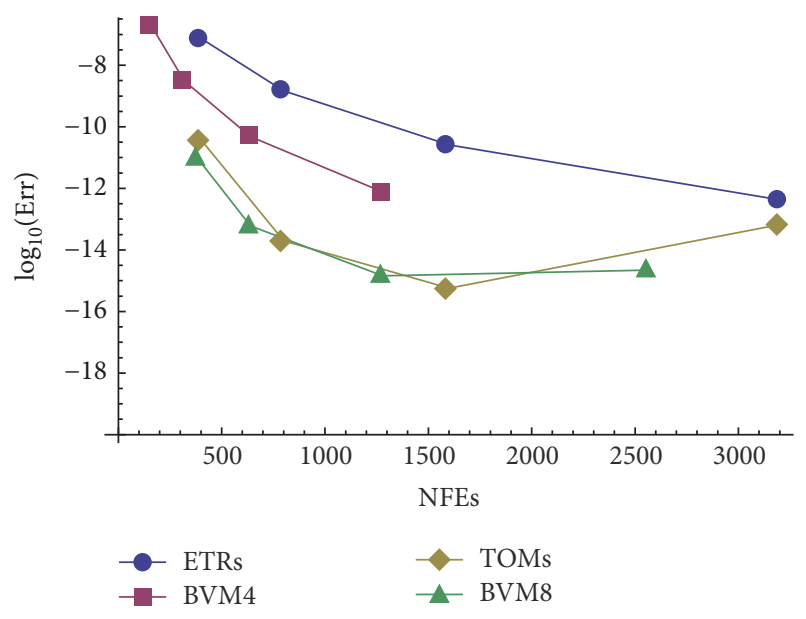

Figure 2: Efficiency curve for Example 2.

these methods are consistent with the theoretical order (order 6) behavior of the methods. We do not calculate the ROC of the TOMs and BVM8 because their errors are mainly due to round-off errors rather than to truncation errors. Figure 2 shows the efficiency curves of these methods.

Example 3. We consider the nonlinear BVP with mixed boundary conditions given in [23]

$$
\begin{aligned}
y^{\prime \prime} & =\frac{\left(y^{\prime}\right)^{2}+y^{2}}{2 e^{x}}, \quad 0<x<1 \\
y(0)-y^{\prime}(0) & =0, \\
y(1)+y^{\prime}(1) & =2 e \\
\text { Exact: } y(x) & =e^{x} .
\end{aligned}
$$

This problem was chosen to demonstrate the performance of the BVMs on nonlinear BVPs with mixed boundary 
TABLE 3: Maximum Errors for different stepsizes for Example 2.

\begin{tabular}{|c|c|c|c|c|c|c|}
\hline$N$ & ETRs [5] & ROC & BVM4 & ROC & TOMs [5] & BVM8 \\
\hline 20 & $7.448 e-08$ & & $1.895 e-07$ & & $3.189 e-11$ & $9.489 e-12$ \\
\hline 40 & $1.480 e-09$ & 5.65 & $3.061 e-09$ & 5.95 & $1.972 e-14$ & $6.021 e-14$ \\
\hline 80 & $2.576 e-11$ & 5.84 & $4.772 e-11$ & 6.00 & $5.722 e-16$ & $1.450 e-15$ \\
\hline 160 & $4.234 e-13$ & 5.93 & $7.538 e-13$ & 5.98 & $6.732 e-14$ & $2.229 e-15$ \\
\hline
\end{tabular}

TABLE 4: Maximum errors for different step sizes for Example 3.

\begin{tabular}{lcccccc}
\hline$N$ & ETRs [5] & ROC & BVM4 & ROC & TOMs [5] & BVM8 \\
\hline 20 & $2.476 e-10$ & & $1.505 e-10$ & & $8.882 e-16$ & $4.441 e-16$ \\
40 & $6.019 e-12$ & 5.36 & $2.347 e-12$ & 6.00 & $4.441 e-16$ & $4.441 e-16$ \\
80 & $6.402 e-14$ & 6.55 & $3.642 e-14$ & 6.01 & $4.441 e-16$ & $4.441 e-16$ \\
160 & $1.010 e-15$ & 5.99 & $6.661 e-16$ & 5.77 & $4.441 e-16$ & $4.441 e-16$ \\
\hline
\end{tabular}

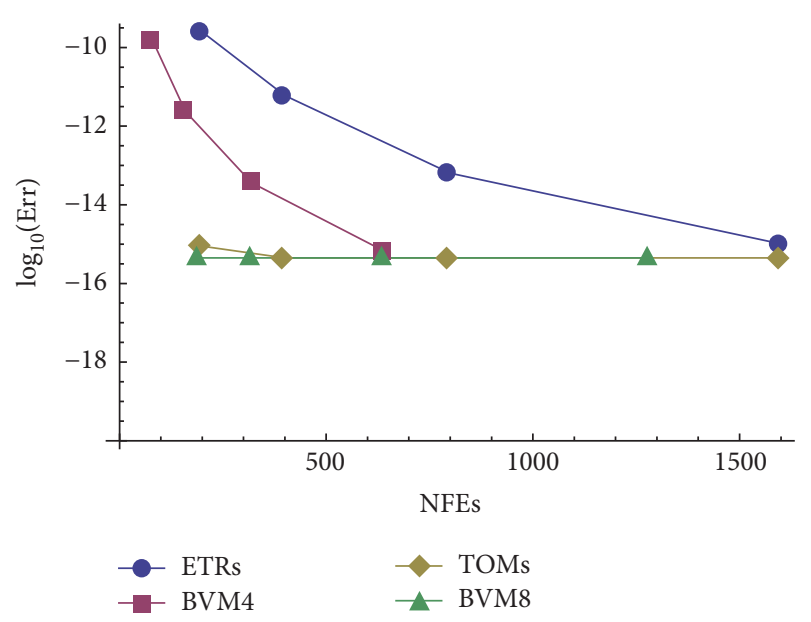

FIgURE 3: Efficiency curve for Example 3.

conditions. The maximum absolute errors were obtained in the range of integration. Table 4 shows the comparison between the ETRs, BVM4, TOMs, and BVM8. Figure 3 shows the efficiency curves of these methods.

Example 4. Consider the second-order BVP given in [24] (bvpT17)

$$
\begin{aligned}
\frac{d^{2} y}{d x^{2}} & =\frac{-3 \lambda y}{\left(\lambda+x^{2}\right)^{2}}, \quad-0.1<x<0.1 \\
y(-0.1) & =\frac{-0.1}{\sqrt{\lambda+0.001}}, \\
y(0.1) & =\frac{0.1}{\sqrt{\lambda+0.001}}
\end{aligned}
$$

Exact: $y(x)=\frac{x}{\sqrt{\lambda+x^{2}}}$.

In order to assess the efficiency of our methods, we solve the boundary layer problem given in [24] (bvpT17). The maximum absolute errors were obtained in the range of integration. Tables 5 and 6 show the comparison between the ETRs, BVM4, TOMs, and BVM8 with $\lambda=1$ and 0.1 , respectively. Figure 4 shows the plot of the solution for values of $\lambda=1,0.1,0.01,0.001$ and the solution has a boundary layer at $x=0$.

Example 5. Consider the second-order BVP given in [24] (bvpT20)

$$
\begin{gathered}
\lambda \frac{d^{2} y}{d x^{2}}=-\left(\frac{d y}{d x}\right)^{2}+1, \quad 0<x<1 \\
y(0)=1+\lambda \log \left(\cosh \left(-\frac{0.745}{\lambda}\right)\right), \\
y(1)=1+\lambda \log \left(\cosh \left(\frac{0.255}{\lambda}\right)\right)
\end{gathered}
$$

$$
\text { Exact: } y(x)=1+\lambda \log \left(\cosh \left(x-\frac{0.745}{\lambda}\right)\right) \text {. }
$$

Also, the efficiency of the scheme is shown by solving the problem given in [24] (bvpT20). The maximum absolute errors were obtained in the range of integration. Tables 7 and 8 show the comparison between the ETRs, BVM4, TOMs, and BVM8 with $\lambda=1$ and 0.1 , respectively. Figure 5 shows the plot of the solution for values of $\lambda=1,0.1,0.01$. From the figure, we see that the solution of the problem has a corner layer at $x=0.745$.

Example 6. We consider the Poisson equation given in [25]

$$
\begin{aligned}
u_{x x}(x, y)+u_{y y}(x, y) & =g(x, y) \quad \text { on } R, \\
u(x, 0) & =u(x, 1), \\
u(x, 2) & =e^{-2 \pi} \sin (\pi x), \\
u(0, y) & =\sin (\pi y), \\
u(1, y) & =e^{\pi} \sin (\pi y),
\end{aligned}
$$


TABLE 5: Maximum Errors for different stepsizes for Example 4 for $\lambda=1$.

\begin{tabular}{|c|c|c|c|c|c|c|}
\hline$N$ & ETRs [5] & ROC & BVM4 & ROC & TOMs [5] & BVM8 \\
\hline 20 & $2.178 e-12$ & & $8.635 e-14$ & & $1.804 e-16$ & $4.163 e-17$ \\
\hline 40 & $3.782 e-14$ & 5.85 & $1.200 e-15$ & 6.17 & $1.457 e-16$ & $4.857 e-17$ \\
\hline 80 & $6.245 e-16$ & 5.92 & $1.110 e-16$ & 3.43 & $3.469 e-17$ & $1.749 e-16$ \\
\hline 160 & $5.551 e-17$ & 3.49 & $3.747 e-16$ & 1.76 & $7.633 e-17$ & $4.163 e-17$ \\
\hline
\end{tabular}

TABLE 6: Maximum Errors for different stepsizes for Example 4 for $\lambda=0.1$.

\begin{tabular}{|c|c|c|c|c|c|c|}
\hline$N$ & ETRs [5] & ROC & BVM4 & ROC & TOMs [5] & BVM8 \\
\hline 20 & $3.559 e-09$ & & $1.201 e-09$ & & $5.038 e-12$ & $5.201 e-14$ \\
\hline 40 & $5.279 e-11$ & 6.07 & $1.820 e-11$ & 6.04 & $2.498 e-13$ & $4.663 e-15$ \\
\hline 80 & $7.931 e-13$ & 6.06 & $2.902 e-13$ & 5.97 & $1.110 e-16$ & $1.110 e-16$ \\
\hline 160 & $1.221 e-14$ & 6.02 & $6.106-15$ & 5.57 & $1.665 e-16$ & $4.163 e-16$ \\
\hline
\end{tabular}

TABLE 7: Maximum Errors for different stepsizes for Example 5 for $\lambda=1$.

\begin{tabular}{|c|c|c|c|c|c|c|}
\hline$N$ & ETRs [5] & ROC & BVM4 & ROC & TOMs [5] & BVM8 \\
\hline 20 & $2.235 e-09$ & & $1.664 e-09$ & & $1.998 e-14$ & $4.452 e-14$ \\
\hline 40 & $3.570 e-11$ & 5.96 & $2.823 e-11$ & 5.88 & $1.776 e-15$ & $4.441 e-15$ \\
\hline 80 & $5.607 e-13$ & 5.99 & $4.370 e-13$ & 6.01 & $6.661 e-16$ & $6.661 e-16$ \\
\hline 160 & $9.104 e-15$ & 5.94 & $6.883 e-15$ & 5.99 & $6.661 e-16$ & $8.882 e-16$ \\
\hline
\end{tabular}

TABle 8: Maximum Errors for different stepsizes for Example 5 for $\lambda=0.1$.

\begin{tabular}{lccccrc}
\hline$N$ & ETRs [5] & ROC & BVM4 & ROC & TOMs [5] & BVM8 \\
\hline 20 & $7.808 e-05$ & & $2.000 e-04$ & & $6.950 e-08$ & $6.527 e-06$ \\
40 & $1.838 e-06$ & 5.41 & $4.090 e-06$ & 5.61 & $4.707 e-06$ \\
80 & $3.163 e-08$ & 5.86 & $5.784 e-08$ & 6.14 & $4.674 e-12$ & $8.212 e-09$ \\
160 & $5.176 e-10$ & 5.93 & $7.066 e-10$ & 6.36 & $2.665 e-15$ & $6.771 e-12$ \\
\hline
\end{tabular}

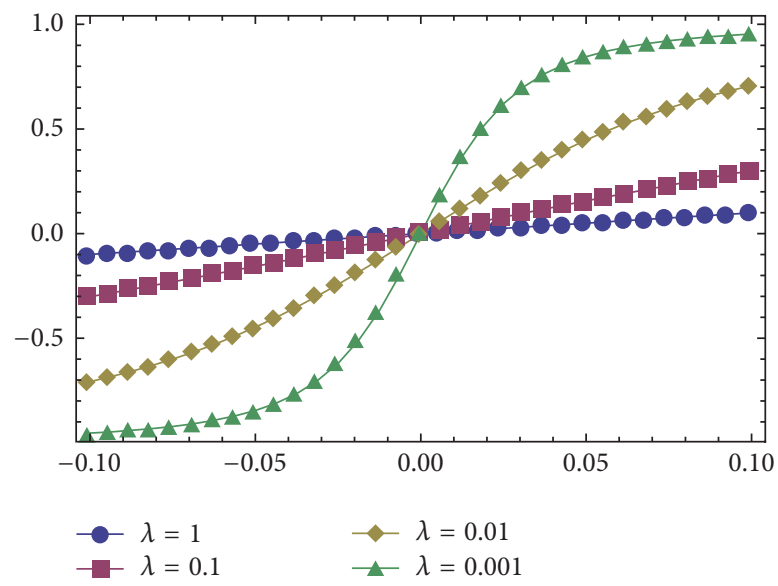

Figure 4: Plot of solution for Example 4.

where $R=\{(x, y): 0 \leq x \leq 1,0 \leq y \leq 2\}$ and $g(x, y)=$ $2 \pi\left(2 \pi y^{2}-2 \pi y-1\right) e^{\pi y(1-y)} \sin (\pi y)$ :

Exact: $u(x, y)=e^{\pi y} \sin (\pi y)+e^{\pi y(1-y)} \sin (\pi x)$.

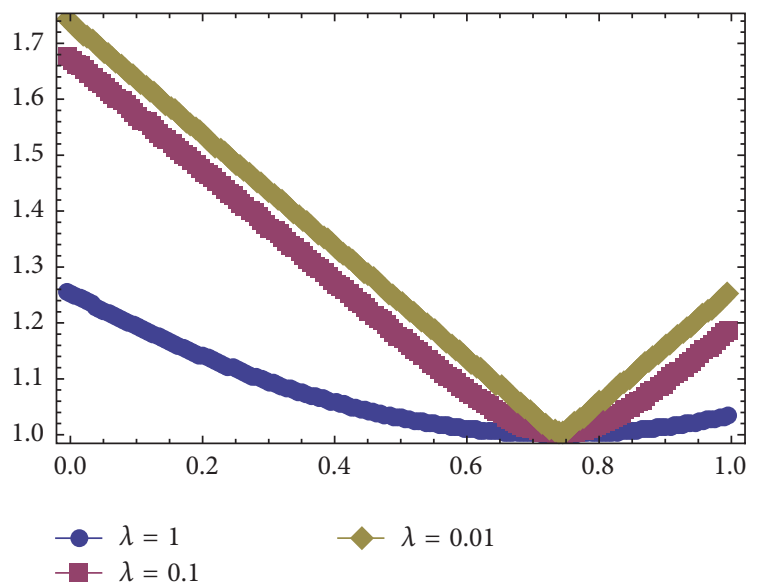

FIGURE 5: Plot of solution for Example 5.

This example shows the performance of the BVMs on the Poisson equation. In order to solve the equation using the BVMs, we carry out the semidiscretization of the spatial variable $x$ using the second-order finite difference method 


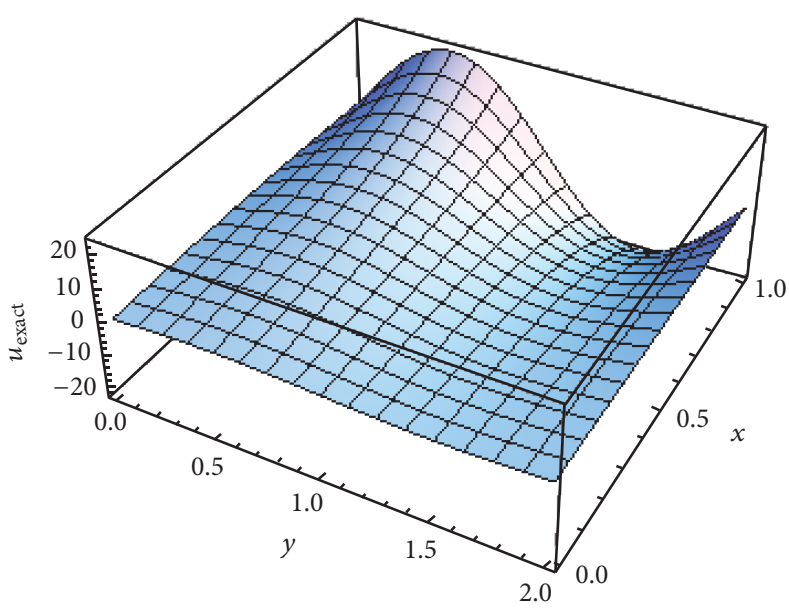

(a) Exact

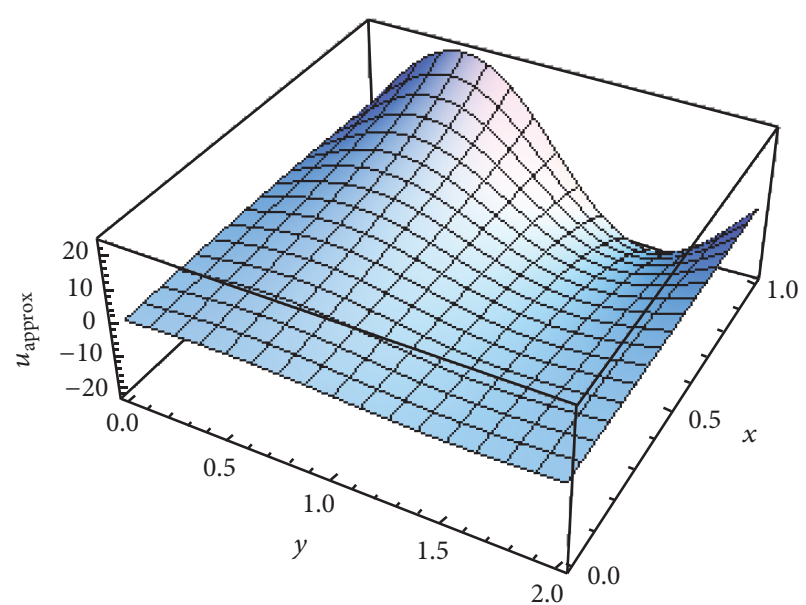

(b) Approximate

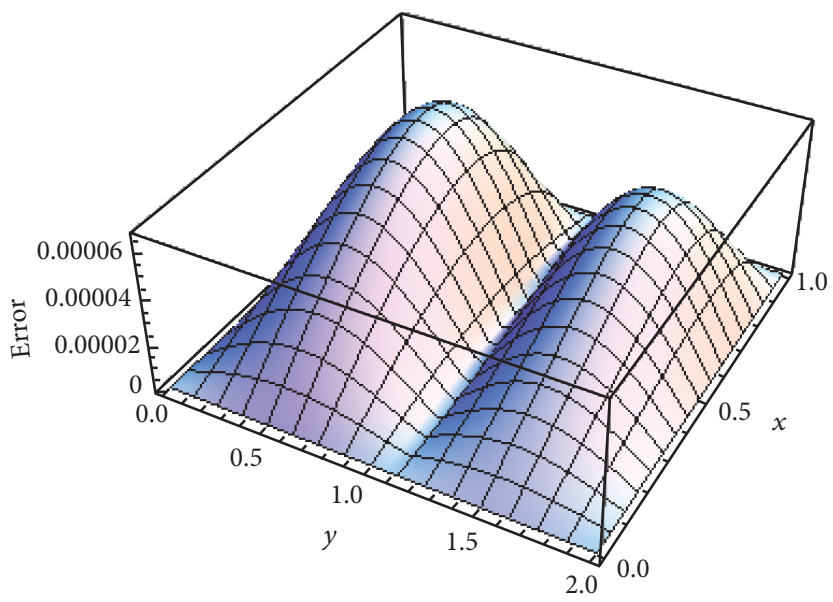

(c) Error function

FIgURe 6: Plot of solution for the Poisson equation.

to obtain the following second-order system in the second variable $y$ :

$$
\begin{aligned}
& \frac{\partial^{2} u_{m}}{\partial y^{2}}+\frac{u_{m+1}(y)-2 u_{m}(y)+u_{m-1}(y)}{(\Delta x)^{2}}=g_{m}(y), \\
& u\left(x_{m}, 0\right)=u\left(x_{m}, 1\right), \\
& u\left(x_{m}, 2\right)=e^{-2 \pi} \sin \left(\pi x_{m}\right),
\end{aligned}
$$

where $\Delta x=(b-a) / M, x_{m}=a+m \Delta x, m=0,1, \ldots, M, \mathbf{u}=$ $\left[u_{1}(y), \ldots, u_{M}(y)\right]^{T}, \mathbf{g}=\left[g_{1}(y), \ldots, g_{M}(y)\right]^{T}, u_{m}(y) \approx$ $u\left(x_{m}, y\right)$, and $g_{m}(y) \approx g\left(x_{m}, y\right)$ which can be written in the form

$$
\mathbf{u}^{\prime \prime}=\mathbf{f}(y, \mathbf{u})
$$

subject to the boundary conditions $\mathbf{u}\left(y_{0}\right)=\mathbf{u}\left(y_{M / 2}\right), \mathbf{u}\left(y_{M}\right)=$ $\mathbf{u}_{M}$, where $\mathbf{f}(y, \mathbf{u})=\mathbf{A u}+\mathbf{g}$ and $\mathbf{A}$ is an $M-1 \times M-1$ matrix arising from the semidiscretized system and $\mathbf{g}$ is a vector of constants. Table 9 shows the comparison between the BVM
TABle 9: Maximum error for the Poisson equation on $y=1$.

\begin{tabular}{lcc}
\hline$h$ & Method in [20] & BVM4 \\
\hline$\frac{1}{16}$ & $3.266 e-02$ & $2.605 e-03$ \\
$\frac{1}{32}$ & $8.210 e-03$ & $3.141 e-04$ \\
$\frac{1}{64}$ & $2.053 e-03$ & $3.947 e-05$ \\
$\frac{1}{128}$ & $5.128 e-04$ & $4.952 e-06$ \\
$\frac{1}{256}$ & - & $6.199 e-07$ \\
\hline
\end{tabular}

and the method in [25]. Figure 6 shows the plot of the exact, approximate, and error function of the problem.

Example 7. Lastly, we consider the Sine-Gordon nonlinear hyperbolic equation given in [26]

$$
u_{y y}(x, y)=u_{x x}(x, y)+\sin (u) \quad \text { on } R
$$




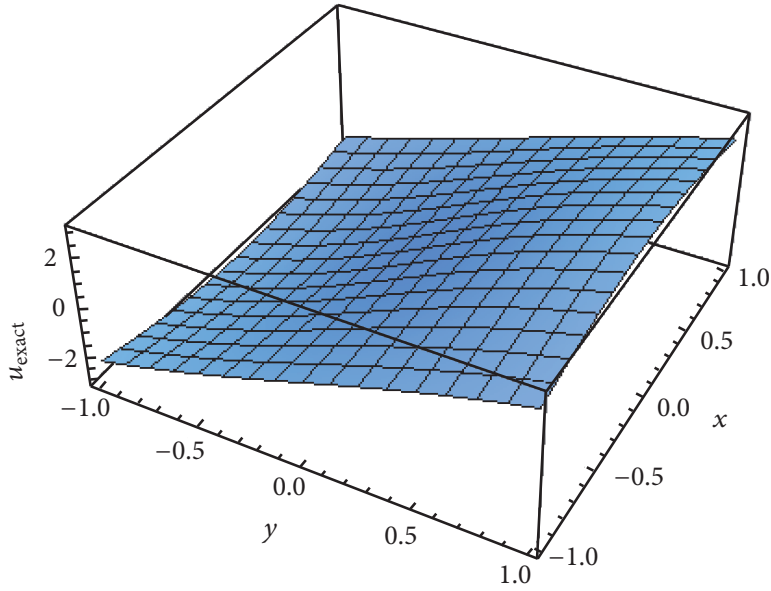

(a) Exact

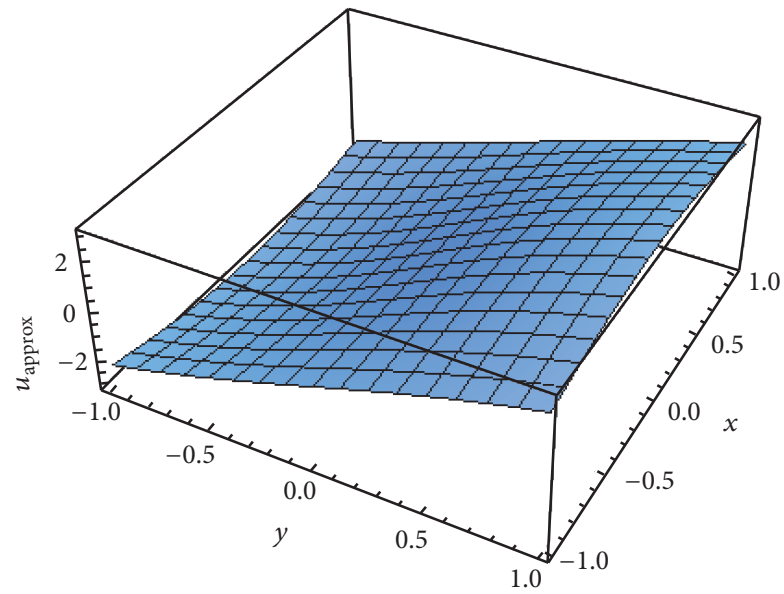

(b) Approximate

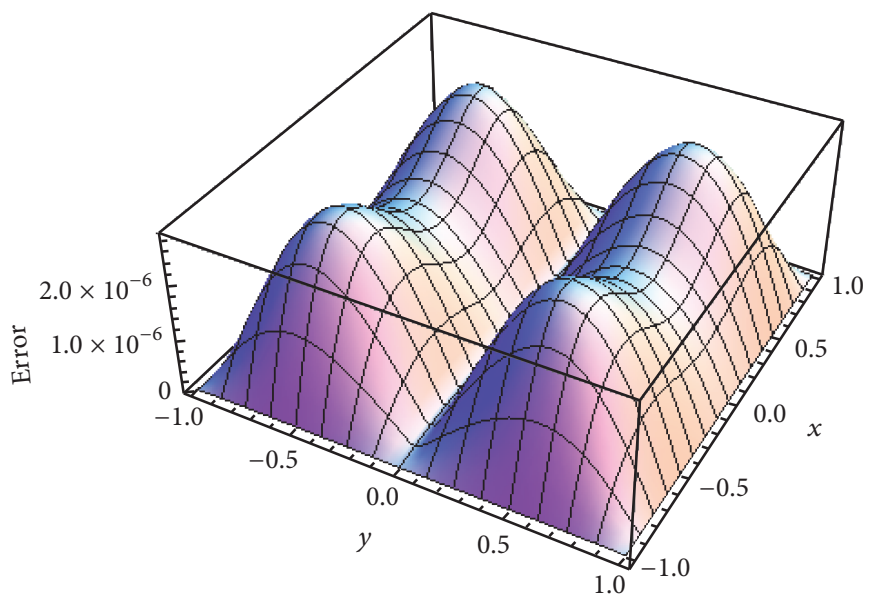

(c) Error function

FIgURE 7: Plot of solution for the Sine-Gordon equation.

subject to the initial conditions

$$
\begin{gathered}
u(x, 0)=0, \\
u_{y}(x, 0)=4 \operatorname{sech}(\pi x),
\end{gathered}
$$

$$
-1 \leq x \leq 1
$$

and the boundary conditions

$$
\begin{aligned}
& u(0, y)=4 \tan ^{-1}(y), \\
& u(1, y)=4 \tan ^{-1}(\operatorname{sech}(1) y),
\end{aligned}
$$

$$
-1 \leq x \leq 1,
$$

where $R=\{(x, y):-1 \leq x \leq 1,-1 \leq y \leq 1\}$. Hence,

$$
\text { Exact: } u(x, y)=4 \tan ^{-1}(\operatorname{sech}(x) y) \text {. }
$$

This example shows the performance of the BVMs on the hyperbolic problem. We discretize the $x$ variable using finite difference schemes to obtain the system

$$
\begin{aligned}
\frac{\partial^{2} u_{m}}{\partial y^{2}}= & \frac{u_{m+1}(y)-2 u_{m}(y)+u_{m-1}(y)}{(\Delta x)^{2}} \\
& +\sin \left(u_{m}(y)\right), \quad m=1, \ldots, M-1, \\
u\left(x_{m}, 0\right)= & 0, \\
u^{\prime}\left(x_{m}, 0\right)= & 4 \operatorname{sech}\left(\pi x_{m}\right),
\end{aligned}
$$

where $\Delta x=(b-a) / M, x_{m}=a+m \Delta x, m=0,1, \ldots, M, \mathbf{u}=$ $\left[u_{1}(y), \ldots, u_{M}(y)\right]^{T}, \mathbf{g}=\left[g_{1}(y), \ldots, g_{M}(y)\right]^{T}, u_{m}(y) \approx$ $u\left(x_{m}, y\right)$, and $g_{m}(y) \approx g\left(x_{m}, y\right)$ which can be written in the form

$$
\mathbf{u}^{\prime \prime}=\mathbf{f}(y, \mathbf{u})
$$

subject to the initial conditions $\mathbf{u}\left(y_{0}\right)=\mathbf{u}_{0}, \mathbf{u}^{\prime}\left(y_{0}\right)=\mathbf{u}_{0}^{\prime}$, where $\mathbf{f}(y, \mathbf{u})=\mathbf{A u}+\mathbf{g}$ and $\mathbf{A}$ is an $M-1 \times M-1$ matrix arising from the semidiscretized system and $\mathbf{g}$ is a vector of constants. Table 10 shows the computational results for this example using the BVM of order 6 . Figure 7 shows the plot of the exact, approximate, and the error function of the problem. 
TABLE 10: Maximum errors for the Sine-Gordon equation.

\begin{tabular}{|c|c|c|c|c|c|}
\hline \multirow{2}{*}{$h$} & 1 & 1 & 1 & 1 & 1 \\
\hline & $\overline{16}$ & $\overline{32}$ & $\overline{64}$ & $\overline{128}$ & $\overline{256}$ \\
\hline & $33.6 e-04$ & $4.7 e-06$ & $6.0 e-07$ & $7.6 e-08$ & $9.6 e-09$ \\
\hline
\end{tabular}

\section{Conclusions}

This paper is concerned with the solution of systems of second-order boundary value problems. This has been achieved by the construction and implementation of a family of BVMs. The methods are applied as a block unification method to obtain the solution on the entire interval of integration. We established the convergence of the methods. We have also shown that the methods are competitive with existing methods cited in the literature.

In the future, we would like to develop a variable step size version of the BVMs with an automatic error estimation.

\section{Competing Interests}

The authors declare that there are no competing interests regarding the publication of this manuscript.

\section{References}

[1] L. Brugnano and D. Trigiante, "On the characterization of stiffness for ODEs," Dynamics of Continuous, Discrete and Impulsive Systems, vol. 2, no. 3, pp. 317-335, 1996.

[2] L. Brugnano, F. Mazzia, and D. Trigiante, "Fifty years of stiffness," in Recent Advances in Computational and Applied Mathematics, T. E. Simos, Ed., pp. 1-21, Springer, 2011.

[3] F. Iavernaro, F. Mazzia, and D. Trigiante, "Stability and conditioning in numerical analysis," Journal of Numerical Analysis, Industrial and Applied Mathematics, vol. 1, no. 1, pp. 91-112, 2006.

[4] L. Brugnano and D. Trigiante, "High-order multistep methods for boundary value problems," Applied Numerical Mathematics, vol. 18, no. 1-3, pp. 79-94, 1995.

[5] L. Brugnano and D. Trigiante, Solving Differential Problems by Multistep Initial and Boundary Value Methods, vol. 6 of Stability and Control: Theory, Methods and Applications, Gordon and Breach Science, Amsterdam, The Netherlands, 1998.

[6] L. Brugnano and D. Trigiante, "Stability properties of some boundary value methods," Applied Numerical Mathematics, vol. 13, no. 4, pp. 291-304, 1993.

[7] P. Amodio and F. Mazzia, "A boundary value approach to the numerical solution of initial value problems by multistep methods," Journal of Difference Equations and Applications, vol. 1, no. 4, pp. 353-367, 1995.

[8] P. Amodio and L. Brugnano, "Parallel implementation of block boundary value methods for ODEs," Journal of Computational and Applied Mathematics, vol. 78, no. 2, pp. 197-211, 1997.

[9] D. O. Awoyemi, "A class of continuous methods for general second order initial value problems in ordinary differential equations," International Journal of Computer Mathematics, vol. 72, no. 1, pp. 29-37, 1999.

[10] J. Vigo-Aguilar and H. Ramos, "Variable stepsize implementation of multistep methods for $y^{\prime \prime}=f\left(x, y, y^{\prime}\right)$," Journal of
Computational and Applied Mathematics, vol. 192, no. 1, pp. 114131, 2006.

[11] J. D. Lambert and I. A. Watson, "Symmetric multistep methods for periodic initial value problems," Journal of the Institute of Mathematics and Its Applications, vol. 18, no. 2, pp. 189-202, 1976.

[12] P. Amodio and F. Iavernaro, "Symmetric boundary value methods for second order initial and boundary value problems," Mediterranean Journal of Mathematics, vol. 3, no. 3-4, pp. 383398, 2006.

[13] T. A. Biala, "A computational study of the boundary value methods and the block unification methods for $y^{\prime \prime}=f\left(x, y, y^{\prime}\right)$," Abstract and Applied Analysis, vol. 2016, Article ID 8465103, 14 pages, 2016.

[14] T. A. Biala and S. N. Jator, "A boundary value approach for solving three-dimensional elliptic and hyperbolic partial differential equations," SpringerPlus Journals, vol. 4, no. 1, article no. 588, 2015.

[15] S. N. Jator and J. Li, "An algorithm for second order initial and boundary value problems with an automatic error estimate based on a third derivative method," Numerical Algorithms, vol. 59, no. 3, pp. 333-346, 2012.

[16] L. Aceto, P. Ghelardoni, and C. Magherini, "PGSCM: a family of P-stable Boundary Value Methods for second-order initial value problems," Journal of Computational and Applied Mathematics, vol. 236, no. 16, pp. 3857-3868, 2012.

[17] P. Onumanyi, U. W. Sirisena, and S. N. Jator, "Continuous finite difference approximations for solving differential equations," International Journal of Computer Mathematics, vol. 72, no. 1, pp. 15-27, 1999.

[18] T. A. Biala, S. N. Jator, and R. B. Adeniyi, "Numerical approximations of second order PDEs by boundary value methods and the method of lines," Afrika Matematika, 2016.

[19] P. L. Ndukum, T. A. Biala, S. N. Jator, and R. B. Adeniyi, "On a family of trigonometrically fitted extended backward differentiation formulas for stiff and oscillatory initial value problems," Numerical Algorithms, vol. 74, no. 1, pp. 267-287, 2017.

[20] M. El-Gamel, "Sinc-collocation method for solving linear and nonlinear system of second-order boundary value problems," Applied Mathematics, vol. 03, no. 11, pp. 1627-1633, 2012.

[21] P. A. Cook, "Estimates for the inverse of a matrix," Linear Algebra and Its Applications, vol. 10, pp. 41-53, 1975.

[22] F. Geng and M. Cui, "Homotopy perturbation-reproducing kernel method for nonlinear systems of second order boundary value problems," Journal of Computational and Applied Mathematics, vol. 235, no. 8, pp. 2405-2411, 2011.

[23] R. S. Stepleman, “Tridiagonal fourth order approximations to general two-point nonlinear boundary value problems with mixed boundary conditions," Mathematics of Computation, vol. 30, pp. 92-103, 1976.

[24] https://archimede.dm.uniba.it/ bvpsolvers/testsetbvpsolvers/ ?page_id=29.

[25] E. A. Volkov, A. A. Dosiyev, and S. C. Buranay, "On the solution of a nonlocal problem," Computers \& Mathematics with Applications, vol. 66, no. 3, pp. 330-338, 2013.

[26] M. Dehghan and A. Shokri, "A numerical method for onedimensional nonlinear sine-Gordon equation using collocation and radial basis functions," Numerical Methods for Partial Differential Equations, vol. 24, no. 2, pp. 687-698, 2008. 


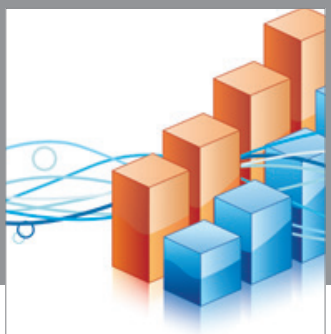

Advances in

Operations Research

vatem alat4

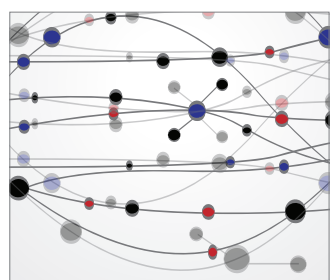

\section{The Scientific} World Journal
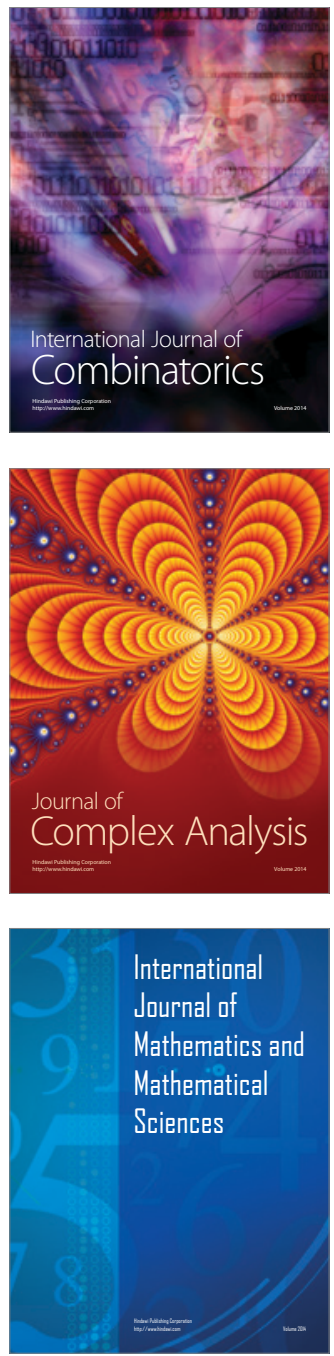
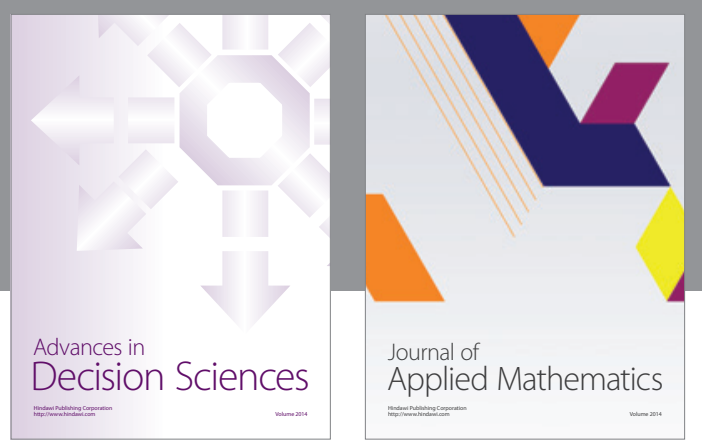

Algebra

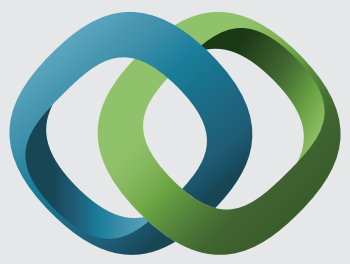

\section{Hindawi}

Submit your manuscripts at

https://www.hindawi.com
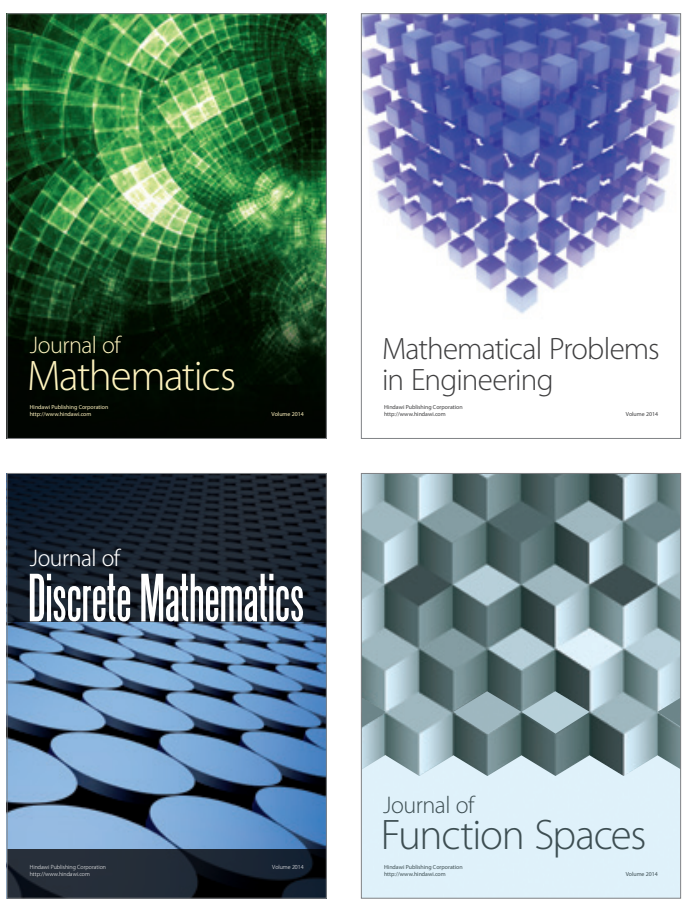

Mathematical Problems in Engineering
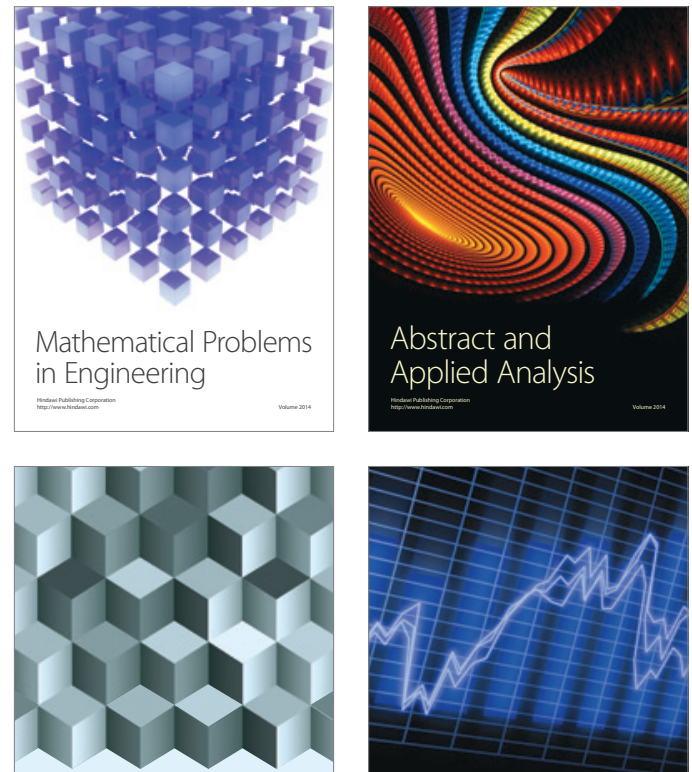

Journal of

Function Spaces

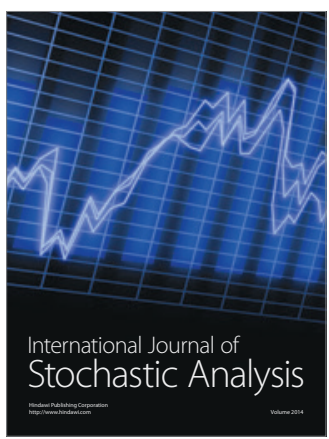

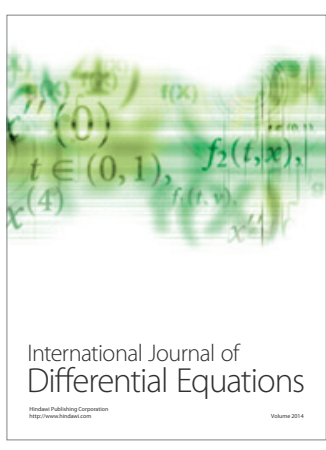
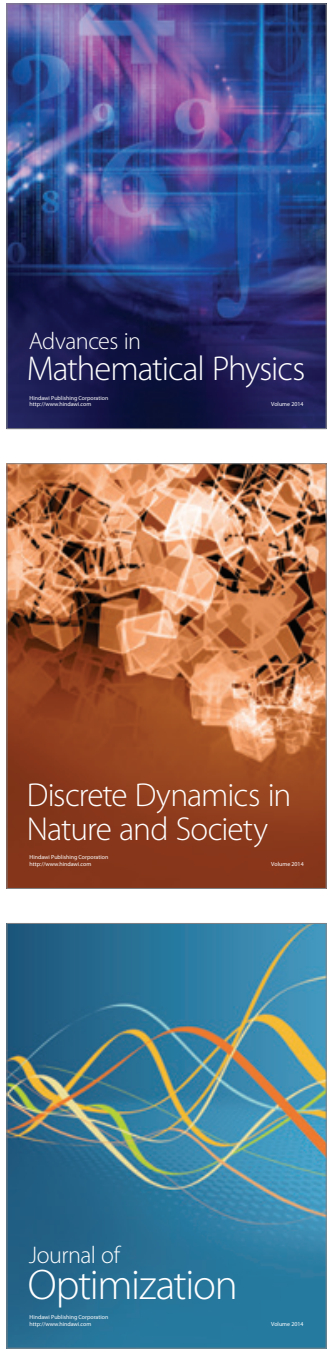